\title{
Trio receives Lasker Foundation Clinical Award for breakthroughs in leukemia treatment
}

When Brian J. Druker was a boy, he wanted to be a baseball player; Nicholas B. Lydon had his sights set on flying jets; Charles L. Sawyers knew early on that he wanted to practice medicine. Decades later, this trio (Figure 1) would collaborate to revolutionize the treatment of chronic myelogenous leukemia (CML). On September 14, the Albert and Mary Lasker Foundation announced that they will recognize these researchers with the 2009 Lasker-DeBakey Clinical Medical Research Award for research that led to the development of drugs, including imatinib (Gleevec) and dasatinib (Sprycel), which have converted CML from a fatal cancer to a manageable condition. Notably, imatinib was the first successful, molecularly targeted, small-molecule drug approved for cancer therapy. The winners spoke with the JCI about their success story.

\section{An oncogenic odyssey}

The understanding of CML pathology and the development of drugs to treat it have unfolded over a half century. CML, which currently affects over 22,000 Americans, is a slow-growing bone marrow cancer resulting in the overproduction of white blood cells. In the early 1960s, Peter Nowell (University of Pennsylvania) and David Hungerford (Fox Chase Cancer Center, Temple University) identified a characteristic small chromosome in the tumor cells of CML patients $(1,2)$, which they designated the Philadelphia chromosome. Over the next two decades, the Philadelphia chromosome was shown to result from a reciprocal translocation between chromosomes 9 and 22 (3), involving parts of the genes V-abl Abelson murine leukemia viral oncogene homolog

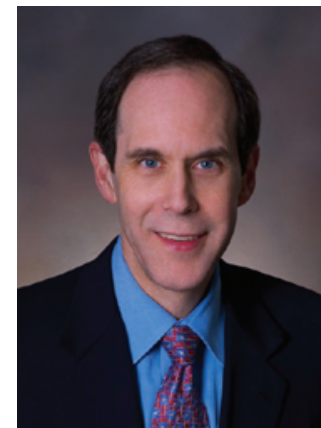

Figure 1

Brian J. Druker, Nicholas B. Lydon, and Charles L. Sawyers have been awarded the 2009 Lasker-DeBakey Clinical Medical Research Award for research that led to the development of drugs, including imatinib (Gleevec) and dasatinib (Sprycel), which revolutionized the treatment of chronic myelogenous leukemia. transformed cells. In 1996, their collaborative efforts demonstrated that imatinib was a potent and specific inhibitor of CML cells in culture and when grown as tumors in mice, and it spared normal cells from damage (10, 11). BCR-ABL wasn't just a requirement for CML cell proliferation; the cells were addicted to it, and without it they died. Imatinib also had pharmacokinetic properties that made it a suitable candidate for development as a therapeutic. There was, however, skepticism in the field that a tyrosine kinase inhibitor would work in CML patients. "We were constantly bombarded with criticism at the time," recalls Lydon. "The view then was that cancer was far more complicated and people didn't believe that targeting a single genetic abnormality would be sufficient. Despite our success in preclinical models, people were doubtful that the drug would reach sufficient concentrations in patient cells and there were concerns about potential toxicity," Druker told the JCI. That same year, Druker was interviewed by an Associated Press reporter accustomed to daily press releases announcing a new cure for cancer. Her interview notes revealed a healthy amount of journalistic skepticism: "nice guy, really good with his patients for a researcher, but that drug is not going anywhere." Druker chuckled as he revealed that the reporter is now his wife. "She still believes I am a nice 1 (ABL1) on chromosome 9 and breakpoint cluster region $(B C R)$ on chromosome 22 (4). The constitutively active tyrosine kinase produced by the $B C R-A B L$ fusion gene stimulates myeloid cell hyperproliferation, the hallmark ofCML(5-9). In the chronic phase of disease, the BCR$\mathrm{ABL}$ translocation arises in a hematopoietic stem cell, and although myeloid lineage cells undergo hyperproliferation, they continue to function normally. With time, CML develops into an acute leukemia known as blast crisis, via an intermediate accelerated phase, which affects myeloid and lymphoid cells. Patients in blast crisis possess additional chromosomal abnormalities, and their granulocyte-macrophage progenitors
OHSU, he also continued treating CML patients, but he was determined to develop a better treatment for the disease without the harmful effects of chemotherapy.

Meanwhile, at Ciba-Geigy Pharmaceuticals Inc., Lydon led a program to identify and develop tyrosine protein kinase inhibitors for use in a number of cancers. The team at Ciba-Geigy identified the ABL inhibitor imatinib (STI571, CGP 57148, Gleevec) in 1992. In what was to become a fortuitous partnership between academia and industry, Druker teamed up with Lydon and others at CibaGeigy, to profile imatinib and its precursor, CGP 53716, in models of CML, with the ultimate goal of blocking the growth of $\mathrm{BCR}-\mathrm{ABL}$ guy and pretty good with my patients."

Novartis (created in 1996 through the merger of Ciba-Geigy and Sandoz) was initially reluctant to develop imatinib for CML, a disease diagnosed in fewer than 5,000 individuals each year. "It's a difficult decision for big pharmaceutical companies to move a candidate drug into clinical trials," Druker explained. "The market for a CML therapy was relatively small and we didn't envisage it being this successful," Lydon said, "but things really changed when we were able to demonstrate the effect of imatinib on ex vivo CML cells, and without damage to normal cells. It was very convincing data." Druker convinced Novartis to move the compound 
into clinical trials and, after a slow start, having the juggernaut of a big pharmaceutical company behind them accelerated clinical testing. "Once Novartis saw imatinib's success, they put their muscle behind the project and it moved with lightning speed into clinical trials," recalled Druker. In June of 1998 , in a collaborative effort led by Druker, and involving teams led by Sawyers (HHMI, now at Memorial Sloan-Kettering Cancer Center but then at UCLA) and Moshe Talpaz (M.D. Anderson Cancer Center), a pill form of imatinib entered phase I clinical trials for the treatment of patients with chronic phase CML who had received prior but failed treatment with the then-current standard of care, the immune system booster IFN- $\alpha$ (12). This trial, in addition to large-scale follow-up phase II and III trials, demonstrated that a daily dose of imatinib was effective in treating chronic phase CML $(13,14)$. Daily-dose therapy returned white blood cell counts to normal, with only minor side effects. The trials led to accelerated FDA approval of imatinib for the treatment of CML on May 10, 2001. Less than three weeks later, imatinib landed on the cover of Time magazine, hailed as the magic bullet in the war against cancer. In an interview with the JCI, Sawyers said, "The year leading up to that point, where many of us were treating well over 100 patients in each of our centers, was the busiest time of my life. We were on a righteous mission to get that drug across the goal line. It was incredibly gratifying to see patients responding well to therapy, but in the midst of it all I never imagined the drug would make the cover of Time. We were just working on our little problem, hoping to make a difference. It was surprising to see the impact on the imagination of the cancer research community. Once the drug worked, the word spread like wildfire. Patients were talking in doctor's waiting rooms and on the Internet, which meant that patient recruitment was never a barrier." Druker remembers that "by that point, we had been in clinical trials for three years and patients were doing incredibly well - surviving and thriving. In my mind it was the most fantastic thing to see the treatment succeed in patients." "Our most optimistic expectations were exceeded beyond our wildest dreams," Lydon recalled.

\section{Many roads to resistance}

Despite the meteoric clinical success of imatinib in controlling CML in chronic phase and subsequently in blast crisis, the longer-term news was not all good. In about $10 \%-15 \%$ of patients, the effects of imatinib were short-lived and patients relapsed and/or progressed to accelerated phase or blast crisis within a year $(13,14)$. "Initially, people literally about to die in the ICU were walking home and playing tennis again within a couple of weeks of starting therapy," recounted Sawyers. "After seeing such a great turnaround in blast crisis patients with full-blown leukemia, the shock of seeing them develop resistance was an emotional rollercoaster for both patients and physicians. We knew that singletarget drugs really don't cure cancer and that eventually there was going to be a dark cloud in an otherwise beautiful story. But it was really rough," he admitted. "We immediately knew that we needed to understand this resistance," acknowledged Druker.

Sawyers' team met this challenge head on, and in 2001 his lab made a major breakthrough: they discovered that imatinib resistance was caused by either a single point mutation in the ABL kinase domain, known to form a critical hydrogen bond with the drug, or by $B C R-A B L$ gene amplification (15). The group, in collaboration with structural biologist John Kuriyan (HHMI, UC Berkeley), went on to discover a spectrum of point mutations that occur at the site of imatinib binding or have a conformational effect on the kinase, such that imatinib is unable to bind to it (16). "John's contribution to the field has been immense," declared Lydon to the JCI. Sawyers recalled that "at the time, I usually didn't read structural biology papers. But we read John's work on the crystal structure of ABL bound to an analog of imatinib and I thought, 'I gotta call this guy.' John was so generous. Within 15 minutes he sent me a Powerpoint slide of a computer model that clearly showed that the T315I mutation was obscuring the binding pocket of the drug. It was incredibly eye opening."

\section{The next generation}

After uncovering an array of imatinib resistance mutations, Sawyers' group sought to develop a second generation of BCR-ABL inhibitors. In collaboration with researchers at Bristol-Myers Squibb, his team showed that the broad-spectrum tyrosine kinase inhibitor dasatinib (Sprycel, BMS-354825), which binds the ABL kinase domain in a manner distinctly different from that of imatinib, retained activity against 14 of 15 imatinib resistance mutations and, in a clinical trial conducted in collaboration with Moshe Talpaz at M.D. Anderson Cancer Center, was able to induce complete and durable hematological responses in chronic phase CML patients with imatinib resistance or intolerance $(17,18)$. Their work suggested that a combination of inhibitors may prevent the emergence of resistant subclones. Dasatinib and the imatinib-derivative nilotinib (Tasigna) are now FDA approved for imatinib-resistant or-intolerant CML (19). Meanwhile, imatinib has now also been approved to treat gastrointestinal stromal tumors (20) and hypereosinophilic syndrome (21), the pathologies of which involve an imatinib-susceptible kinase. Given that oncogenic kinases are at the root of a number of major malignancies, such as non-small cell lung cancer, acute myeloid leukemia, polycythemia vera, renal cancer, and breast cancer, it is possible that imatinib and its analogs will be used to treat other cancers.

When asked whether there is another imatinib out there, all three responded with a hearty "yes." "There might not be another drug that as a single, daily pill causes a $95 \%$ remission rate that lasts for years, but there is a growing number of drugs that target mutant kinases, such as gefitinib (Iressa) and erlotinib (Tarceva), and produce dramatic responses in other cancers. These drugs will change the way those cancers are treated," said Sawyers. On the FDA approval process, he commented that "the FDA is not necessarily tougher today. Compelling data is compelling data." Druker echoed that sentiment: "When there is high medical need, the FDA can react quickly. The medical research community needs to develop more effective drugs, not ones with a month or two of benefit; that's not what the FDA is looking for."

\section{The final frontier}

Thanks to Druker, Lydon, and Sawyers, and their respective research teams, what was once an invariably fatal illness within five years of diagnosis is now a chronic but manageable condition. However, while tyrosine kinase inhibitors are able to render CML largely inactive during treatment, neither imatinib nor its derivatives are effective in CML patients who carry the T315I BCR-ABL mutation. Nor have these drugs been able to eliminate the residual leukemic stem cell that carries the translocation. "We have spent eight years trying to identify a compound to circumvent this kinase mutation. I call it the recalcitrant mutation," lamented Druker. "It's a thorn in the field's side," acknowledged Sawyers, "but I believe it will be removed in a year or two. A lot of efforts are under way to find a T315I inhibitor and it has been more challenging than people first thought." Malignancies are no longer classified solely on histology, but are now being reclassified according to 
their genotypes, with the hope of developing molecularly targeted therapies. "It's funny that almost 10 years later some people are just now recognizing how important genetic mutations are in predicting response to therapy. It takes time for a field to 'get it,' "' said Sawyers. "Now, the notion of oncogene addiction is catchy and sexy and has become commonplace; in many ways, imatinib's development established that paradigm.”

Eradicating CML at the stem cell level and determining the substrates key for stem cell renewal are added hurdles on the horizon. Lydon, who now works primarily as a consultant for Ambit Biosciences and AnaptysBio asserted, "We need to be able to differentiate between normal stem cells and early foundational cancer stem cells, and how the latter can persist, despite therapy. We also have to devise combinations of drugs that block different and independent tumorigenesis signaling pathways." Druker added, "If we can keep patients on chronic therapy, without long-term side effects, then this is a huge advance. But admittedly, they would rather be cured; they want a treatment that will allow them to get off all therapies forever."

\section{Partnership is the key}

The development of successful treatments for CML is rooted in over four decades of discovery and development in fields as diverse as molecular oncology and structural biology and speaks to the value of a multidisciplinary approach to conquering disease. "Breakthroughs don't come about without lots of different areas of investigation converging," insisted Druker, adding that he considers Lydon a scientific soulmate. "We shared a common vision - to get a tyrosine kinase inhibitor into the clinic. Nick was always willing to share data and was a true scientific collaborator. The partnerships between academia and industry have gotten much more difficult in the last 10 years and drug development has become a much more lengthy process." Lydon reiterated the value of their multidisciplinary alliance: "It was incredibly good to collaborate with someone who was not just a scientist but was also a physician, and had that understanding of translational research. At that time, I was naive about the clinical aspects of CML. Having that partner able to translate the data into the clinic was very important." Sawyers echoed these sentiments: "When working on figuring out resistance mechanisms, it was really energizing to have a group of structural biologists and physician scientists together in a room, first trying to teach each other what we were doing and why we were doing it, and then seeing the data come together. You have to have an open mind. It is to John Kuriyan's credit that he took my phone call and was willing to teach me what he knew."

Given the landmark discoveries of these three researchers, the reactions of their families are curious. "I probably still embarrass my 11-year-old in front of his friends, but he does understand that I've made an important contribution to medicine," said Druker. "In her kindergarten class, my 6-year-old drew a picture of her parents and beneath it wrote, 'My Dad is an important doctor.' My wife and I kind of cringed at that." Sawyers mused, "I think my kids are impressed; they like talking about what I do in the lab. But beyond sentence number 3 they are back to whatever Game Boy thing they are playing. My daughter took freshman biology last year and they were studying oncogenes and tumor suppressor genes, so I took the opportunity to tell her the imatinib story." When Druker and Lydon were asked whether they had any regrets about straying from their respective early aspirations on the baseball diamond and in aeronautics, it was no surprise to hear them declare, "Absolutely none."

\section{The luster of the Lasker}

Since 1945, the Albert and Mary Lasker Foundation Awards Program has recognized the contributions of physicians, scientists, and public servants who have made major advances in the understanding, diagnosis, treatment, cure, and prevention of human disease. The winners will receive their awards at a luncheon in New York City on October 2 and will share a \$250,000 honorarium. All three awardees were quick to express what an extraordinary honor and surprise the recognition was and noted that they share this honor with the teams of scientists that were an integral part of this endeavor.

Each recipient was reluctant to speculate that the Lasker Awards are often a precursor to the Nobel Prize, and each stressed that their patients, not prizes, drive their commitment. "A large part of the reward is my relationships with our patients, who I got to know incredibly well. Many from the first trial are still alive and have become incredible cancer advocates," said Sawyers. Druker agreed: "The reality is that I get to go to the clinic every week and see patients benefiting from this incredible work. I keep pictures of all of my patients and it is my connection with them that is the best award."

\section{Brooke Grindlinger}

1. Nowell, P., and Hungerford, D. 1960. A minute chromosome in human chronic granulocytic leukemia [abstract]. Science. 132:1497.

2. Nowell, P.C., and Hungerford, D.A. 1961. Chromosome studies in human leukemia. II. Chronic granulocytic leukemia. J. Natl. Cancer Inst. 27:1013-1035.

3. Rowley, J.D. 1980. Ph1-positive leukemia, including chronic myelogenous leukemia. Clin. Haematol. 9:55-86.

4. Groffen, J., et al. 1984. Philadelphia chromosomal breakpoints are clustered within a limited region, bcr, on chromosome 22. Cell. 36:93-99.

5. Collins, S.J., Kubonishi, I., Miyoshi, I., and Groudine, M.T. 1984. Altered transcription of the c-abl oncogenes in K-562 and other chronic myelogenous leukemia cells. Science. 225:72-74.

6. Gale, R.P., and Canaani, E. 1984. An 8-kilobase abl RNA transcript in chronic myelogenous leukemia. Proc. Natl. Acad. Sci. U.S.A. 81:5648-5652.

7. Shtivelman, E., Lifshitz, B., Gale, R.P., and Canaani, E. 1985. Fused transcript of abl and bcr genes in chronic myelogenous leukemia. Nature. 315:550-554.

8. Ben-Neriah, Y., Daley, G.Q., Mes-Masson, A.M., Witte, O.N., and Baltimore, D. 1986. The chronic myelogenous leukemia-specific P210 protein is the product of the bcr/abl hybrid gene. Science. 233:212-214.

9. Davis, R.L., Konopka, J.B., and Witte, O.N. 1985. Activation of the c-abl oncogene by viral transduction or chromosomal translocation generates altered c-abl proteins with similar in vitro kinase properties. Mol. Cell. Biol. 5:204-213.

10. Druker, B.J., et al. 1996. Effects of a selective inhibitor of Abl tyrosine kinase on the growth of $\mathrm{Bcr}-\mathrm{Abl}$ positive cells. Nat. Med. 2:561-566.

11. Carroll, M., et al. 1997. CGP 57148, a tyrosine kinase inhibitor, inhibits the growth of cells expressing BCR-ABL, TEL-ABL, and TEL-PDHGR fusion proteins. Blood. 90:4947-4952.

12. Druker, B.J., et al. 2001. Efficacy and safety of a specific inhibitor of the BCR-ABL tyrosine kinase in chronic myeloid leukemia. N. Engl. J. Med. 344:1031-1037.

13. Druker, B.J., et al. 2001. Activity of a specific inhibitor of the BCR-ABL tyrosine kinase in the blast crisis of chronic myelogenous leukemia and acute lymphoblastic leukemia with the Philadelphia chromosome. N. Engl. J. Med. 344:1038-1042.

14. Sawyers, C.L., et al. 2002. Imatinib induces hematologic and cytogenetic responses in patients with chronic myelogenous leukemia in myeloid blast crisis: results of a phase II study. Blood. 99:3530-3539.

15. Gorre, M.E., et al. 2001. Clinical resistance to STI571 cancer therapy caused by BCR-ABL gene mutation or amplification. Science. 293:876-880.

16. Shah, N.P., et al. 2002. Multiple BCR-ABL kinase domain mutations confer polyclonal resistance to the tyrosine kinase inhibitor imatinib (STI571) in chronic phase and blast crisis chronic myeloid leukemia. Cancer Cell. 2:117-125.

17. Shah, N.P., et al. 2004. Overriding imatinib resistance with a novel ABL kinase inhibitor. Science. 305:399-401.

18. Talpaz, M., et al. 2006. Dasatinib in imatinib-resistant Philadelphia chromosome-positive leukemias. N. Engl. J. Med. 354:2531-2541.

19. Kantarjian, H., et al. 2006. Nilotinib in imatinibresistant CML and Philadelphia chromosome-positive ALL. N. Eng. J. Med. 354:2542-2551.

20. Demetri, G.D., et al. 2002. Efficacy and safety of imatinib mesylate in advanced gastrointestinal stromal tumors. N. Engl. J. Med. 347:472-480.

21. Gleich, G.J., et al. 2002. Treatment of hypereosinophilic syndrome with imatinib mesilate. Lancet. 359:1577-1578. 